\title{
Early results after operatively versus non-operatively treated flail chest: a retrospective study focusing on outcome and complications
}

\author{
Mathieu M. E. Wijffels ${ }^{1} \cdot$ Tjebbe Hagenaars $^{1} \cdot$ Diba Latifi $^{1}$ - Esther M. M. Van Lieshout ${ }^{1} \cdot$ Michael H. J. Verhofstad $^{1}$
}

Received: 11 December 2017 / Accepted: 29 April 2018 / Published online: 21 May 2018

(c) The Author(s) 2018

\begin{abstract}
Purpose Flail chest was traditionally treated non-operatively using mechanical ventilation and pain control. In order to reduce the occurrence of ventilation-associated complications and long-term disability, operative rib fixation is becoming a proven standard therapy for these patients. However, the consequences of the surgical complications may influence success rates negatively. The aim of this study was to compare the outcome of flail chest treatment by surgical rib fixation with nonoperative treatment, with special focus on the impact of surgical complications.

Methods A retrospective case series of operatively treated flail chest patients was compared with non-operatively treated patients. Patients' injury and treatment characteristics and outcome parameters (e.g., duration of mechanical ventilation, length of Intensive Care stay (ICLOS) and hospital length of stay (HLOS), mortality, surgery-related complications and pneumonia) were collected from the patients' medical files. Crude and matched-pairs analyses were performed in SPSS.

Results Twenty-three operatively and 47 non-operatively treated patients were enrolled. Operatively treated patients required significantly shorter mechanical ventilation; median 4 days versus 12 days for the non-operative group $(p=0.011)$. The matched-pairs analysis also showed a lower pneumonia rate $(35 \%$ versus $80 \%$; $p=0.035$ ) and a shorter HLOS (median 21 versus 23 days; $p=0.028$ ) in the operative group. No significant differences in duration of ICLOS, and occurrence of other injury-related adverse events were found between both groups. Seven surgery-related complications occurred, of which three required invasive solutions.

Conclusions Operative fixation of a flail chest in trauma patients results in a lower rate of pneumonia, less mechanical ventilation days and shorter hospital stay, compared with non-operative treatment, but at the cost of surgery-related complications requiring invasive solutions in some cases.
\end{abstract}

Keywords Flail chest $\cdot$ Operative $\cdot$ Outcome $\cdot$ Pneumonia $\cdot$ Rib fracture $\cdot$ Complications

\section{Introduction}

Chest wall injury is common following blunt trauma $[1,2]$. Flail chest is among the most severe chest wall injuries. A flail chest is defined as three or more consecutive rib fractures, in two or more places, creating a flail segment [3, 4]. This is the most severe form of rib fractures and this injury is associated with significant morbidity and mortality rates [5]. Patients suffering from flail chest are traditionally treated non-operatively and require mechanical ventilation

Mathieu M. E. Wijffels

m.wijffels@erasmusmc.nl

1 Trauma Research Unit, Department of Surgery, Erasmus MC, University Medical Center Rotterdam, P.O. Box 2040, 3000 CA Rotterdam, The Netherlands and pain control resulting in prolonged stay at the Intensive Care Unit (ICU) [6]. Prolonged ventilation is associated with ventilation-related, potentially lethal complications such as pneumonia, adult respiratory distress syndrome, and sepsis $[7,8]$. In addition, long-term morbidities such as pain, disability, respiratory complications, and inability to resume work have been described $[9,10]$.

Nowadays, a surgical approach for this injury by fixating the ribs is increasingly applied and might offer a decrease in morbidity and mortality rates. Metal plates, intramedullary devices, and encircling wires are implants that are designed to increase chest wall stability supporting the healing process and thereby correction of chest wall deformity [11-14]. More and more evidence shows the benefits of surgical fixation of flail chests over non-operative management [15-18]. Although recent evidence-based guidelines advise surgical 
fixation in flail chest patients $[19,20]$, the benefit of rib fixation is not unanimous and needs more comparative studies $[21,22]$. However surgical techniques may overcome conservative complications, it may introduce the risk of surgical side-effects. To our knowledge no studies focused extensively on these complications and the required solutions.

In our institution, surgical rib fixation has been a part of the treatment protocol for flail chest since March 2011. This protocol aims to fixate the flail chest operatively within $48 \mathrm{~h}$ after hospital admission, to optimize the lung capacity and to reduce the duration of mechanical ventilation, the occurrence of post-traumatic pneumonia and the ICU length of stay.

The aim of this study was to compare the outcome of flail chest treatment by surgical rib fixation with non-operative treatment, with special focus on the impact of surgical complications. Primary outcome measure is the occurrence of pneumonia. Secondary outcome measures included the total hospital length of stay, the duration of mechanical ventilation, surgical complications and the ICU length of stay.

\section{Patients and methods}

\section{Population}

Between March 1, 2011 and March 31, 2014, most patients admitted to a level I trauma center with the diagnosis flail chest have been treated operatively. During this period, surgical rib fixation was the standard-of-care treatment for this injury. Hemodynamically unstable patients or those with a bad or infaust neurological prognosis were treated conservatively for their flail chest, at the consent of the attending surgeon. A historic control group, suffering from flail chest treated non-operatively, at the same hospital between October 1, 2006 and October 31, 2010, was selected. Patients in the control group were identified based upon a text search in the radiology reports and discharge letters. Flail chest was defined as paradoxical movement of the thoracic wall during breathing or three or more adjacent ribs fractured in two or more places as found on the computed tomography (CT). The CT scan made on admission was reviewed by one of investigators $(\mathrm{TH})$ and the research assistant (DL) in order to confirm the diagnosis flail chest and to determine the number of fractured ribs. Patients for whom CT scans were not available, who were transferred to another hospital for treatment, or who died within $24 \mathrm{~h}$ after hospital admission were excluded. The medical research ethics committee exempted this study.

\section{Treatment}

Depending on the clinical situation, patients were admitted to the ICU or the clinical ward for monitoring and supportive treatment. Non-operative treatment for patients with flail chest consisted of oxygen support or positive pressure mechanical ventilation for respiratory support, guided by the patients' oxygen saturation and analgesia for pain control. Individual optimal saturation was determined by the attending ICU-doctor or surgeon. Analgesia included oral medication, intravenous medication, or epidural catheters. Bronchodilators and mucolytics were inhaled at a maximum of six times a day. In the postoperative care, no differences can be found between the surgical and non-surgical treatment protocol.

Operative treatment for patients with flail chest was performed by the attending trauma surgeon. The MatrixRIB (DePuy Synthes, West Chester, Pennsylvania), consisting of pre-contoured metal locking plates, locking screws, and intramedullary splints, was used for the internal fixation of the ribs. A lateral or posterior muscle sparing approach was used. Surgical aim was to stabilize at least one fracture site per rib of the flail segment. Single fractured ribs were not fixated, unless dislocated, as judged by the operating trauma surgeon during the operation. Post-operatively, patients received a chest tube and analgesia for pain control. Postoperative care was provided at the ICU if needed or the clinical ward. Chest tubes were removed when there was no air leak, no pneumothorax on chest radiograph and drainage was less than $100 \mathrm{ml}$ per $24 \mathrm{~h}$.

During the inclusion period selective decontamination of the digestive tract was introduced on the ICU for those patients with an expected duration of mechanical ventilation longer than $48 \mathrm{~h}$.

\section{Outcome and data collection}

The primary outcome, pneumonia, was defined by fever $\left(>38.5{ }^{\circ} \mathrm{C}\right.$ ), positive mucus culture, and prescribed antibiotics focused on pulmonary organisms. Secondary outcomes included days of mechanical ventilation, ICU length of stay, hospital length of hospital stay, and other adverse events. Outcome data were collected retrospectively from the patient's medical files during the first 30 days after trauma. Patient, injury-related, and surgery-related characteristics were collected from the patient's medical files. Surgical complications were noted in the patient files and were defined as "adverse events in recovery, attributable to the surgery performed for rib fixation". 


\section{Statistical analysis}

Analyses were performed using the Statistical Package for the Social Sciences (SPSS, version 21, SPSS Inc., Chicago, IL, USA). Normality of continuous data was checked using the Shapiro-Wilk test, and homogeneity of variance across groups was determined using the Levene's test. Baseline characteristics, fracture characteristics, and outcome of patients treated operatively were compared with those of the control group. Continuous data, which were all nonparametric, are presented as medians with first and third quartile. Categorical variables are provided as numbers and percentages. In the crude analysis, data were compared using a Mann-Whitney $U$ test (continuous data) or a Chi-squared test (categorical data).

In order to study the effect of operative treatment more specifically, a matched pair analysis was performed. A matched non-operatively treated control was searched for each operatively treated patient. Controls were considered adequate if they were treated non-operatively, had a comparable age ( $\leq 10$ years difference), identical gender, comparable ASA score (American Society of Anesthesiologists classification; $\leq 1$ difference), and a comparable ISS (within the same group of ISS $<25$ versus ISS $>25$ ). Controls were selected with replacement; therefor the use of a single control for multiple cases was allowed. Groups were compared using a Wilcoxon signed-rank test (continuous data) or a McNemar test (categorical data). Results with $p<0.05$ (twosided test) were regarded as statistically significant.

\section{Results}

\section{Patient and injury characteristics}

During the study period, all 23 operatively treated and 47 non-operatively treated patients were enrolled in the operative and non-operative treatment group, respectively. No data were available on those patients conservatively treated in the "operation period". Concerning all included patients the median age was 51 years, $52(74 \%)$ were male and 36 (53\%) had ASA class I (Table 1). Almost all injuries $(N=68$; 97\%) occurred after a blunt, high energy trauma of which 67 patients (96\%) had a unilateral flail segment. Fifty-seven patients $(81 \%)$ had radiological signs of lung contusion adjacent to the flail chest. ISS score was 32 and 31 in the non-operatively and operatively treated group, respectively $(p=0.477)$.
Table 1 Characteristics for the entire study population separated by treatment $(N=70)$

\begin{tabular}{|c|c|c|c|c|}
\hline & Overall $(N=70)$ & $\begin{array}{l}\text { Non-operative } \\
\text { treatment }(N=47)\end{array}$ & $\begin{array}{l}\text { Operative treat- } \\
\text { ment }(N=23)\end{array}$ & $P$ value \\
\hline \multicolumn{5}{|l|}{ Patients' characteristics } \\
\hline Age at trauma (years) ${ }^{\mathrm{a}}$ & $51(40-66)$ & $49(40-63)$ & $60(40-69)$ & $0.268^{\mathrm{c}}$ \\
\hline Male gender ${ }^{\mathrm{b}}$ & $52(74 \%)$ & $37(79 \%)$ & $15(65 \%)$ & $0.354^{\mathrm{d}}$ \\
\hline \multicolumn{5}{|l|}{$\mathrm{ASA}^{\mathrm{b}}$} \\
\hline ASA-I & $36(53 \%)$ & $27(60 \%)$ & $9(39 \%)$ & $0.171^{\mathrm{e}}$ \\
\hline ASA-II & $21(31 \%)$ & $13(29 \%)$ & $8(35 \%)$ & \\
\hline ASA-III & $11(16 \%)$ & $5(11 \%)$ & $6(26 \%)$ & \\
\hline \multicolumn{5}{|l|}{ Injury characteristics } \\
\hline High energy trauma ${ }^{\mathrm{b}}$ & $68(97 \%)$ & $45(96 \%)$ & $23(100 \%)$ & $0.895^{\mathrm{d}}$ \\
\hline \multicolumn{5}{|l|}{ Affected side flail segment ${ }^{b}$} \\
\hline Unilateral & $67(96 \%)$ & $46(98 \%)$ & $21(91 \%)$ & $0.499^{\mathrm{d}}$ \\
\hline Bilateral & $3(4 \%)$ & $1(2 \%)$ & $2(9 \%)$ & \\
\hline Lung contusion ${ }^{\mathrm{b}}$ & $57(81 \%)$ & $37(79 \%)$ & $20(87 \%)$ & $0.628^{\mathrm{d}}$ \\
\hline Unilateral & $45(79 \%)$ & $30(81 \%)$ & $15(75 \%)$ & $0.829^{\mathrm{d}}$ \\
\hline Bilateral & $12(21 \%)$ & $7(19 \%)$ & $5(25 \%)$ & \\
\hline ISS $^{\mathrm{a}}$ & $33(23-41)$ & $34(24-41)$ & $29(20-41)$ & $0.462^{\mathrm{c}}$ \\
\hline GCS on admission ${ }^{\mathrm{a}}$ & $14(3-15)$ & $14(3-15)$ & $14(10-15)$ & $0.502^{\mathrm{c}}$ \\
\hline Intubated on admission $^{\mathrm{a}}$ & $20(29 \%)$ & $15(32 \%)$ & $5(22 \%)$ & $0.554^{\mathrm{d}}$ \\
\hline Total number of fractured ribs ${ }^{a}$ & $10(8-12)$ & $9(8-11)$ & $10(9-14)$ & $0.130^{\mathrm{c}}$ \\
\hline Fractured ribs at flail segment ${ }^{\mathrm{a}}$ & $5(4-7)$ & $5(4-6)$ & $6(4-7)$ & $0.148^{\mathrm{c}}$ \\
\hline Fractured ribs at non-flail side ${ }^{a}$ & $5(3-7)$ & $4(2-7)$ & $5(3-7)$ & $0.435^{\mathrm{A}}$ \\
\hline
\end{tabular}

Data are presented as ${ }^{\mathrm{a}}$ median $\left(\mathrm{P}_{25}-\mathrm{P}_{75}\right)$ or as ${ }^{\mathrm{b}}$ number $(\%)$ and were analyzed with a ${ }^{\mathrm{c}}$ Mann-Whitney $U$ test, ${ }^{\mathrm{d}}$ Fisher's exact test, or a ${ }^{\mathrm{e}} \mathrm{Chi}$-squared analysis

ASA American Society of Anesthesiologists, GCS Glasgow Coma Scale, HET High Energy Trauma, ISS Injury Severity Score 
The median number of fractured ribs per patient was ten, five at the flail segment and five at the contralateral side. No statistically significant differences were found in baseline characteristics when comparing the two groups.

Of the 23 operatively treated patients, 15 underwent surgical rib fixation within $24 \mathrm{~h}$, an additional 6 between 24 and $48 \mathrm{~h}$ and all within $96 \mathrm{~h}$ after hospital admission (Table 2). The median duration of anesthesia and surgery was $171 \mathrm{~min}$. A median number of four ribs was fixated. Locking plates were used in $88 \%$ of the fixated ribs, and were preferred over intramedullary splints. No intra-operative complications occurred.

\section{Crude analysis}

The rate of pneumonia did not differ statistically significant between the two groups ( $35 \%$ in the operative group versus $57 \%$ in the non-operative group; $p=0.126$; Table 3 ). When comparing surgically treated patients receiving/not receiving SDD, no differences in the occurrence of pneumoniae were found (respectively, $7 / 16$ versus $1 / 7, p=0.172$ ). When comparing conservatively treated patients receiving/not receiving SDD, no differences in the occurrence of pneumoniae were found (respectively, $12 / 24$ versus $11 / 18, p=0.431$ ).

Most patients were directly admitted to the ICU $(89 \%$ versus $91 \% ; p=1.000)$, and five $(7 \%)$ patients in total were readmitted to the ICU (4 versus $1 ; p=0.932$ ). Although the median cumulative ICLOS was twice as long in the nonoperative group (10 days versus 5 days in the operative group), this did not reach statistical significance $(p=0.254)$.

The percentage of patients requiring mechanical ventilation was similar in both groups (62 versus $78 \% ; p=0.264$ ). Overall, the operative group required shorter mechanical ventilation than the non-operative group (4 days versus 12 days; $p=0.011$ ). No statistical differences were found in the number of patients who received SDD when comparing the surgically treated and conservatively treated patients $(70 \%$ vs. $51 \%, p=0.162$ )

Table 2 Treatment characteristics for the operative group $(N=23)$

\begin{tabular}{lc}
\hline & $\begin{array}{c}\text { Operative treat- } \\
\text { ment }(N=23)\end{array}$ \\
\hline${\text { Duration of surgery }(\mathrm{min})^{\mathrm{a}}}^{\mathrm{a}}$ & $171(123-203)$ \\
Fixated ribs & $4(4-5)$ \\
Rib fixation & \\
Single fixation & $4(3-5)$ \\
Double fixation $_{\text {Plate fixation }}{ }^{\mathrm{b}}$ & $0(0-2)$ \\
Number of plates $^{\mathrm{a}}$ & $12(52 \%)$ \\
Splints $^{\mathrm{a}}$ & $4(3-5)$ \\
\hline
\end{tabular}

Data are presented as ${ }^{\mathrm{a}}$ median $\left(\mathrm{P}_{25}-\mathrm{P}_{75}\right)$ or as ${ }^{\mathrm{b}}$ number $(\%)$
The median HLOS was 20 days for the operative group versus 23 days for the non-operatively treated group; $p=0.495$.

Adverse events occurred in 12 patients (52\%) of the operative group versus $36(77 \%)$ in the non-operative group, yet this $25 \%$ difference did not reach statistical significance $(p=0.075)$. Overall, six patients died in hospital; two (9\%) in the operative group versus four $(9 \%)$ in the non-operative group $(p=1.000)$. One patient in each group deceased due to the direct effects of flail chest, i.e., pulmonary insufficiency. The other four patients deceased due to traumatic neurological injuries.

Surgery-related adverse events occurred in seven patients $(30 \%)$. Most common surgery-related complications are the surgical site infections, which present approximately 3 weeks after operation with pus. These abcesses needed to be drained, due to fever and radiological signs of fluid collections without other clear reasons for fever. Additional antibiotics were given in two cases; one with signs of sternal osteitis after a sternotomie for CABG in the past, continuous with the abcess. The other one to prevent from infection of nearby spondylodesis material. The third infected wound was treated successfully with negative wound therapy after drainage.

One of the hardware-related complications was splintperforation of the visceral facing cortex. In a second case crosslinking two adjacent ribs occurred. In both patients conservative therapy was successful. Two patients showed bleeding out of the surgical wound which has been treated by local wound care. No systematic or (surgical or radiological) interventions were needed.

\section{Matched-pairs analysis}

Of the 23 operated patients, 20 were successfully matched to a non-operatively treated control patient (Table 4). Of the 20 controls, two were randomly picked twice. Baseline characteristics between groups were comparable, except for the median number of fractured ribs at the flail segment; five ribs in the non-operatively treated group versus 6 ribs in the operatively treated group $(p=0.021)$. No significant differences were found comparing conservatively treated patients excluded from matching with operatively or conservatively treated patients included in the matching process.

The operatively treated group showed a statistically significantly lower pneumonia rate (35 versus $80 \% ; p=0.035$ ) and a lower median HLOS (21 versus 23 days; $p=0.028$ ). (Tables 5, 6) The median number of cumulative ventilation days was lower in the operative group (4 versus 18 days overall; $p=0.012)$. Surgery-related adverse events occurred in six patients $(30 \%)$. 
Table 3 Outcome for the entire study population separated by treatment $(N=70)$

\begin{tabular}{|c|c|c|c|c|}
\hline & Overall $(N=70)$ & $\begin{array}{l}\text { Non-operative } \\
\text { treatment } \\
(N=47)\end{array}$ & $\begin{array}{l}\text { Operative } \\
\text { treatment } \\
(N=23)\end{array}$ & $P$ value \\
\hline HLOS (days) $^{\mathrm{a}}$ & $21(14-32)$ & $23(14-35)$ & $20(13-30)$ & $0.495^{\mathrm{f}}$ \\
\hline First IC admission ${ }^{b}$ & $63(90 \%)$ & $42(89 \%)$ & $21(91 \%)$ & $1.000^{\mathrm{g}}$ \\
\hline Primary ICLOS (days) ${ }^{\mathrm{a}}$ & $7(3-18)$ & $10(3-20)$ & $5(4-11)$ & $0.296^{\mathrm{f}}$ \\
\hline IC readmission ${ }^{\mathrm{b}}$ & $5(7 \%)$ & $4(9 \%)$ & $1(4 \%)$ & $0.932^{\mathrm{g}}$ \\
\hline Secondary ICLOS (days) ${ }^{a}$ & $4(3-10)$ & $5(3-13)$ & $2(2-2)$ & $0.157^{\mathrm{f}}$ \\
\hline Cumulative ICLOS (days) ${ }^{\mathrm{a}}$ & $8(3-18)$ & $10(3-21)$ & $5(4-11)$ & $0.254^{\mathrm{f}}$ \\
\hline Primary mechanical ventilation $^{\mathrm{b}}$ & $47(67 \%)$ & $29(62 \%)$ & $18(78 \%)$ & $0.264^{\mathrm{g}}$ \\
\hline Primary mechanical ventilation (days) ${ }^{\mathrm{a}, \mathrm{c}}$ & $6(3-14)$ & $7(5-17)$ & $4(2-9)$ & $\mathbf{0 . 0 3 1} 1^{\mathrm{f}}$ \\
\hline Re-intubation ${ }^{\mathrm{b}}$ & $5(7 \%)$ & $5(11 \%)$ & $0(0 \%)$ & $0.253^{\mathrm{g}}$ \\
\hline Secondary mechanical ventilation (days) ${ }^{\mathrm{a}}$ & $9(5-25)$ & $9(5-25)$ & NA & NA \\
\hline Cumulative mechanical ventilation (days) ${ }^{\mathrm{a}}$ & $7(3-17)$ & $12(6-18)$ & $4(2-9)$ & $\mathbf{0 . 0 1 1}^{\mathrm{f}}$ \\
\hline Chest tube $^{\mathrm{b}}$ & $62(89 \%)$ & $39(83 \%)$ & $23(100 \%)$ & $0.666^{\mathrm{g}}$ \\
\hline Chest tube (days) $)^{\mathrm{a}, \mathrm{d}}$ & $7(5-9)$ & $7(5-9)$ & $6(5-9)$ & $0.649^{\mathrm{f}}$ \\
\hline Adverse event ${ }^{\mathrm{b}}$ & $48(69 \%)$ & $36(77 \%)$ & $12(52 \%)$ & $0.075^{\mathrm{g}}$ \\
\hline \multicolumn{5}{|l|}{ Surgery-related adverse events } \\
\hline Surgical site infection ${ }^{b}$ & $3(4 \%)$ & NA & $3(13 \%)$ & NA \\
\hline Crosslinking ribs ${ }^{\mathrm{b}}$ & $1(1 \%)$ & NA & $1(4 \%)$ & NA \\
\hline Splint through cortex ${ }^{\mathrm{b}}$ & $1(1 \%)$ & NA & $1(4 \%)$ & NA \\
\hline Bleeding $^{\mathrm{b}}$ & $2(3 \%)$ & NA & $2(9 \%)$ & NA \\
\hline \multicolumn{5}{|l|}{ General adverse events } \\
\hline Pneumonia $^{\mathrm{b}}$ & $35(50 \%)$ & $27(57 \%)$ & $8(35 \%)$ & $0.126^{\mathrm{g}}$ \\
\hline Respiratory insufficiency ${ }^{\mathrm{b}}$ & $7(10 \%)$ & $6(13 \%)$ & $1(4 \%)$ & $0.517^{\mathrm{g}}$ \\
\hline Empyema $^{\text {b }}$ & $1(1 \%)$ & $1(2 \%)$ & $0(0 \%)$ & $1.000^{\mathrm{g}}$ \\
\hline Delirium $^{\mathrm{b}}$ & $20(29 \%)$ & $17(36 \%)$ & $3(13 \%)$ & $0.076^{\mathrm{g}}$ \\
\hline Overall in-hospital mortality ${ }^{\mathrm{b}}$ & $6(9 \%)$ & $4(9 \%)$ & $2(9 \%)$ & $1.000^{\mathrm{g}}$ \\
\hline Injury-related in-hospital mortality ${ }^{\mathrm{b}}$ & $2(33 \%)$ & $1(25 \%)$ & $1(50 \%)$ & $1.000^{\mathrm{g}}$ \\
\hline
\end{tabular}

Data are presented as ${ }^{\mathrm{a}}$ median $\left(\mathrm{P}_{25}-\mathrm{P}_{75}\right)$ or as ${ }^{\mathrm{b}}$ number $(\%)$ and were analyzed with a ${ }^{\mathrm{f}}$ Mann-Whitney $U$ test, ${ }^{g}$ Fisher's exact test. Boldface fonts indicate statistically significant differences

Data missing for ${ }^{\mathrm{c}}$ three, ${ }^{\mathrm{d}}$ four

ICLOS intensive care length of stay, HLOS hospital length of stay, NA not applicable

\section{Discussion}

This comparative study showed that operative fixation of a flail chest in trauma patients accounts for a lower rate of pneumonia, less mechanical ventilation days, and shorter hospital stay at the cost of surgery-related adverse events in $30 \%$ of the patients.

Rib fractures are common and still have a high morbidity and mortality, dependent on the age and number of fractures, with long-lasting complaints [10, 23]. This stresses the need for new and better treatment modalities. The hypothesis on the beneficial effect of surgical rib fixation is twofold. First, stability of the thoracic wall is improved and as a consequence the residual volume is minimized during spontaneous breathing. This will diminish the pneumonia rate and improve oxygenation resulting in a faster recovery. Furthermore, the indicated pain score after surgical fixation is low enabling productive cough, deep breaths, and movement, protecting from pneumonia and other immobility-related complications [24].

Three randomized controlled trials (RCT) have been published describing only 61 operatively treated patients, compared with 62 non-operatively treated patients [13, 25]. Although based on small numbers of included patients, the results are unanimous; operative fixation of rib fractures reduces the duration of ventilation requirement, lower rate of pneumonia, and shorter ICU and hospital stay. Leinicke et al. concluded in a meta-analysis (including two of the three mentioned RCTs), based on nine studies, that operative fixation of flail chest is beneficial in several fields [16]. However, based on 538 patients, only one study in this metaanalysis was rated as high quality. Therefore, case-control studies, such as the current study, are valuable to evaluate daily clinical practice. 
Table 4 Characteristics for the matched pairs separated by treatment $(N=40)$

\begin{tabular}{|c|c|c|c|}
\hline & $\begin{array}{l}\text { Non-operative treatment } \\
(N=20)\end{array}$ & $\begin{array}{l}\text { Operative treatment } \\
(N=20)\end{array}$ & $P$ value \\
\hline \multicolumn{4}{|l|}{ Patients' characteristics } \\
\hline Age at trauma (years) ${ }^{\mathrm{a}}$ & $57(44-69)$ & $60(41-69)$ & $0.247^{\mathrm{c}}$ \\
\hline Male gender ${ }^{\mathrm{b}}$ & $15(75 \%)$ & $15(75 \%)$ & $1.000^{\mathrm{d}}$ \\
\hline \multicolumn{4}{|l|}{$\mathrm{ASA}^{\mathrm{b}}$} \\
\hline ASA-I & $7(35 \%)$ & $8(40 \%)$ & $0.126^{\mathrm{d}}$ \\
\hline ASA-II & $12(60 \%)$ & $7(35 \%)$ & \\
\hline ASA-III & $1(5 \%)$ & $5(25 \%)$ & \\
\hline \multicolumn{4}{|l|}{ Injury characteristics } \\
\hline High energy trauma ${ }^{b}$ & $20(100 \%)$ & $20(100 \%)$ & $1.000^{\mathrm{d}}$ \\
\hline \multicolumn{4}{|l|}{ Affected side flail segment ${ }^{b}$} \\
\hline Unilateral & $20(100 \%)$ & $18(90 \%)$ & $0.500^{\mathrm{d}}$ \\
\hline Bilateral & $0(0 \%)$ & $2(10 \%)$ & \\
\hline Lung contusion $^{\mathrm{b}}$ & $14(70 \%)$ & $18(90 \%)$ & $0.289^{\mathrm{d}}$ \\
\hline Unilateral & $13(93 \%)$ & $13(72 \%)$ & $0.500^{\mathrm{d}}$ \\
\hline Bilateral & $1(7 \%)$ & $5(28 \%)$ & \\
\hline ISS $^{\mathrm{a}}$ & $32(21-41)$ & $31(21-48)$ & $0.477^{\mathrm{c}}$ \\
\hline GCS on admission $^{\mathrm{a}}$ & $13(3-15)$ & $15(6-15)$ & $0.243^{c}$ \\
\hline Intubated on admission $^{\mathrm{a}}$ & $6(30 \%)$ & $5(25 \%)$ & $1.000^{\mathrm{d}}$ \\
\hline Total number of fractured ribs ${ }^{a}$ & $10(8-10)$ & $10(9-14)$ & $0.097^{\mathrm{c}}$ \\
\hline Fractured ribs at flail segment ${ }^{\mathrm{a}}$ & $5(3-7)$ & $6(5-8)$ & $0.021^{\mathrm{c}}$ \\
\hline Fractured ribs at non-flail side ${ }^{a}$ & $5(2-7)$ & $5(3-7)$ & $0.840^{\mathrm{c}}$ \\
\hline
\end{tabular}

Data are presented as ${ }^{\text {a }}$ median $\left(\mathrm{P}_{25}-\mathrm{P}_{75}\right)$ or as ${ }^{\mathrm{b}}$ number $(\%)$ and were analyzed with a ${ }^{\mathrm{c}}$ Wilcoxon signed rank test or a ${ }^{\mathrm{d}} \mathrm{McNemar}$ test

ASA American Society of Anesthesiologists, GCS Glasgow Coma Scale, HET High Energy Trauma, ISS Injury Severity Score
Operative fixation of flail chest seems promising but at the cost of surgery-related complications. In our study, $30 \%$ of the patients suffered from morbidity directly related to surgery. Granetzny et al. report adverse events directly related to surgery in two of the 20 included patients. Marasco et al. and Tanaka et al. do not mention the surgical complications $[13,25,26]$. In our series, three out of seven patients needed invasive techniques to solve the complication. This should be taken into account in counselling the patients suitable for rib fixation. Furthermore, it may be valuable to include the number of surgery-related complications in treatment consensus papers, as published by Pierracci (19). Despite this high number of surgery-related complications, the protocol in our hospital did not change based on these adverse numbers. The enthusiasm for splinting decreased over the years and by and large every rib fixation is carried out with plates only. Furthermore, minimizing of the incision, muscle sparing techniques and a low threshold to leave a wound drain postoperatively became more popular.

Sarani et al. focusses in their paper on the pitfalls of surgical rib fixation, some of which were found in our case series as well [27]. The larger number of surgical complications in our study may be influenced negatively by definition; crosslinking and splints through the cortex stabilizes the thoracic cage in a not-intended way and was there for scored as a complication in our study. Surgery-related complications of $30 \%$ are high, yet without the need of a reintervention; the impact for the patient is negligible. Fixation of the ribs as soon as the patient is cleared for surgery is favorable with regard to better outcome [11, 28]. Unfortunately, the number of surgically treated patients in our study was too small for a reliable evaluation of surgical timing.

Selective decontamination of the digestive tract is proven to protect from infectious complications like pneumoniae [29] however this effect was not found in our study. Pneumoniae rate was independent on the administration of SDD during ICU stay. Most probably this is due to the small number of patients. The role of SDD in this flail chest population remains unknown since most patients remained on mechanical ventilation longer than $48 \mathrm{~h}$ and received SDD in most cases. Larger studies are needed to investigate this role, especially in those patients with concomitant lung contusion.

This study had a number of limitations. First, the short follow-up may have influenced the number of complications. The number of surgical complications may increase after a longer follow-up, since some patients will suffer from symptomatic fixation materials. On the other hand, some non-operatively treated patients will suffer from 
Table 5 Outcome for the matched pairs separated by treatment $(N=40)$

\begin{tabular}{|c|c|c|c|}
\hline & $\begin{array}{l}\text { Non-operative treat- } \\
\text { ment }(N=20)\end{array}$ & $\begin{array}{l}\text { Operative treatment } \\
(N=20)\end{array}$ & $P$ value \\
\hline HLOS (days) $)^{\mathrm{a}}$ & $23(17-42)$ & $21(12-33)$ & $0.028^{c}$ \\
\hline Primary IC admission ${ }^{\mathrm{b}}$ & $19(95 \%)$ & $19(95 \%)$ & $1.000^{\mathrm{d}}$ \\
\hline Primary ICLOS (days) ${ }^{\mathrm{a}}$ & $12(3-29)$ & $5(3-13)$ & $0.131^{\mathrm{c}}$ \\
\hline IC readmission ${ }^{\mathrm{b}}$ & $0(0 \%)$ & $1(5 \%)$ & $1.000^{\mathrm{d}}$ \\
\hline Secondary ICLOS (days) ${ }^{\mathrm{a}}$ & $17(17-17)$ & 7 (N.D.) & N.A \\
\hline Cumulative ICLOS (days) $^{\mathrm{a}}$ & $12(3-29)$ & $5(3-13)$ & $0.172^{\mathrm{c}}$ \\
\hline Primary mechanical ventilation ${ }^{\mathrm{b}}$ & $11(55 \%)$ & $17(85 \%)$ & $0.070^{\mathrm{d}}$ \\
\hline Primary mechanical ventilation (days) ${ }^{\mathrm{a}, \mathrm{e}}$ & $17(9-22)$ & $4(2-10)$ & $\mathbf{0 . 0 2 1} 1^{\mathrm{c}}$ \\
\hline Re-intubation ${ }^{\mathrm{b}}$ & $2(80 \%)$ & $0(0 \%)$ & NA \\
\hline Secondary mechanical ventilation (days) ${ }^{\mathrm{a}}$ & 25 (N.D.) & ND & NA \\
\hline Cumulative mechanical ventilation (days) ${ }^{\mathrm{a}}$ & $18(12-26)$ & $4(2-10)$ & $\mathbf{0 . 0 1 2} 2^{\mathrm{c}}$ \\
\hline Chest tube (N patients) ${ }^{\mathrm{a}}$ & $15(75 \%)$ & $20(100 \%)$ & $0.063^{\mathrm{d}}$ \\
\hline Chest tube (days) ${ }^{\mathrm{a}, \mathrm{f}}$ & $9(5-9)$ & $6(5-9)$ & $0.282^{\mathrm{c}}$ \\
\hline Adverse event ${ }^{\mathrm{b}}$ & $18(90 \%)$ & $11(55 \%)$ & $0.065^{\mathrm{d}}$ \\
\hline \multicolumn{4}{|l|}{ Surgery-related adverse events } \\
\hline Surgical site infection & NA & $2(10 \%)$ & NA \\
\hline Crosslinking ribs & NA & $1(5 \%)$ & NA \\
\hline Splint through cortex & NA & $1(5 \%)$ & NA \\
\hline Bleeding & NA & $2(10 \%)$ & NA \\
\hline \multicolumn{4}{|l|}{ General adverse events } \\
\hline Pneumonia & $16(80 \%)$ & $7(35 \%)$ & $\mathbf{0 . 0 3 5}^{\mathrm{d}}$ \\
\hline Respiratory insufficiency & $0(0 \%)$ & $1(5 \%)$ & $1.000^{\mathrm{d}}$ \\
\hline Empyema & $0(0 \%)$ & $0(0 \%)$ & NA \\
\hline Delirium & $7(35 \%)$ & $3(15 \%)$ & $0.289^{\mathrm{d}}$ \\
\hline Overall in-hospital mortality ${ }^{\mathrm{b}}$ & $1(5 \%)$ & $2(10 \%)$ & $1.000^{\mathrm{d}}$ \\
\hline Injury-related in-hospital mortality ${ }^{\mathrm{b}}$ & $0(0 \%)$ & $1(50 \%)$ & $1.000^{\mathrm{d}}$ \\
\hline
\end{tabular}

Data are presented as ${ }^{\text {a } m e d i a n ~}\left(\mathrm{P}_{25}-\mathrm{P}_{75}\right)$ or as ${ }^{b}$ number $(\%)$ and were analyzed with a ${ }^{c}$ Wilcoxon signed ranks test or a ${ }^{\mathrm{d}} \mathrm{McNemar}$ test. Boldface fonts indicate statistically significant differences

Data missing for ${ }^{\mathrm{e}}$ two, ${ }^{\mathrm{f}}$ three

CLOS intensive care length of stay, HLOS hospital length of stay, NA not applicable

Table 6 Surgery-related complications with treatment modality

\begin{tabular}{llll}
\hline Surgery-related complication & $\begin{array}{l}\text { Presentation (postopera- } \\
\text { tive day) }\end{array}$ & Treatment & Extra information \\
\hline Incisional bleeding 1 & $1 \mathrm{st}$ & Local treatment & Local treatment \\
Incisional bleeding 2 & $1 \mathrm{st}$ & Conservative & On first postoperative X-ray \\
Crosslinking ribs & $1 \mathrm{st}$ & Conservative & Drainage and negative pressure \\
Splint through cortex & $7 \mathrm{th}$ & therapy & On CT due to dyspnea and tachypnea \\
Surgical site infection 1 & 20 th & Drainage and antibiotics & Sternal osteitis due to rib fixation \\
& 26 th & & after sternotomy in past medical \\
history
\end{tabular}

symptomatic rib non-unions [30]. Second, due to its retrospective character, data are based on medical record information that may be incomplete and inaccurate. Comparison with a historical group may introduce unknown bias due to changes in clinical practice over time. Furthermore, no data were available on the non-operatively treated patients during 
the "operation-period". Third, most of the included patients were multi-trauma patients. Therefore, outcome parameters may be influenced by other injuries resulting in less comparable data between groups despite equal ISS score. Fourth, the definition of pneumonia varies widely in rib fixation literature. Therefore, it is hard to compare the outcome of this study with others. Despite these limitations, the scientific evidence favoring surgical stabilization of flail chests has been expanded, with more insight in the accompanying surgical complications.

\section{Conclusion}

From these data, it can be concluded that operative fixation of a flail chest in trauma patients results in a lower rate of pneumonia, less mechanical ventilation days, and shorter hospital stay as compared with non-operative treatment, but at the cost of surgery-related complications.

\section{Compliance with ethical standards}

Conflict of interest All authors declare that they have no conflict of interest.

Research involving human participants All procedures performed in studies involving human participants were in accordance with the ethical standards of the institutional and/or national research committee and with the 1964 Helsinki declaration and its later amendments or comparable ethical standards. For this type of study formal consent is not required.

Open Access This article is distributed under the terms of the Creative Commons Attribution 4.0 International License (http://creativeco mmons.org/licenses/by/4.0/), which permits unrestricted use, distribution, and reproduction in any medium, provided you give appropriate credit to the original author(s) and the source, provide a link to the Creative Commons license, and indicate if changes were made.

\section{References}

1. Ziegler DW, Agarwal NN. The morbidity and mortality of rib fractures. J Trauma. 1994;37(6):975-9.

2. Cameron P, Dziukas L, Hadj A, Clark P, Hooper S. Rib fractures in major trauma. Aust N Z J Surg. 1996;66(8):530-4.

3. Zhang X, Guo Z, Zhao C, Xu C, Wang Z. Management of patients with flail chest by surgical fixation using claw-type titanium plate. J Cardiothorac Surg 2015;10:145.

4. Bergeron E, Lavoie A, Clas D, Moore L, Ratte S, Tetreault S, Lemaire J, Martin M. Elderly trauma patients with rib fractures are at greater risk of death and pneumonia. J Trauma. 2003;54(3):478-85.

5. Ciraulo DL, Elliott D, Mitchell KA, Rodriguez A. Flail chest as a marker for significant injuries. J Am Coll Surg. 1994;178(5):466-70.
6. Simon B, Ebert J, Bokhari F, Capella J, Emhoff T, Hayward T III, Aurelio R. Smith L. Practice management guideline for pulmonary contusion-flail chest. East Practice management Workgroup for pulmonary Contusion-flail chest. J Trauma. 2012;73(5):S351-S361.

7. Pepe PE, Hudson LD, Carrico CJ. Early application of positive end-expiratory pressure in patients at risk for the adult respiratorydistress syndrome. N Engl J Med. 1984;311(5):281-6.

8. Montgomery AB, Stager MA, Carrico CJ, Hudson LD. Causes of mortality in patients with the adult respiratory distress syndrome. Am Rev Resp Dis. 1985;132(3):485-9.

9. Fabricant L, Ham B, Mullins R, Mayberry J. Prolonged pain and disability are common after rib fractures. Am J Surg. 2013;205(5):511-5.

10. Marasco S, Lee G, Summerhayes R, Fitzgerald M, Bailey M. Quality of life after major trauma with multiple rib fractures. Injury. 2015;46(1):61-5.

11. Althausen PL, Shannon S, Watts C, Thomas K, Bain MA, Coll D, O'mara TJ, Bray TJ. Early surgical stabilization of flail chest with locked plate fixation. J Orthop Trauma. 2011;25(11):641-7.

12. Moreno De La Santa Barajas P, Polo Otero MD, Delgado SánchezGracián C, Lozano Gómez M, Toscano Novella A, Leal Ruiloba J, Choren Duran S, Del Prado CM, Maria L. Surgical fixation of rib fractures with clips and titanium bars (STRATOS System). Preliminary experience. Cir Esp. 2010;88(3):180-6.

13. Tanaka H, Yukioka T, Yamaguti Y, Shimizu S, Goto H, Matsuda $\mathrm{H}$, Shimazaki S. Surgical stabilization or internal pneumatic stabilization? A prospective randomized study of management of severe flail chest patients. J Trauma. 2002;52(4):727-32.

14. Paris F, Tarazona V, Blasco E, Canto A, Casillas M, Pastor J, Paris M, Montero R. Surgical stabilization of traumatic flail chest. Thorax. 1975;30:521-7.

15. Cataneo AJ, Cataneo DC, de Oliveira FH, de Oliveira KA, El Dib R, de Oliveira Carvalho PE. Surgical versus nonsurgical interventions for flail chest. Cochrane Database Syst Rev. 2015;29(7):CD009919.

16. Leinicke JA, Elmore L, Freeman BD, Colditz GA. Operative management of rib fractures in the setting of flail chest: A systematic review and meta analysis. Ann Surg. 2013;258(6):914-21.

17. Slobogean GP, MacPherson CA, Sun T, Pelletier ME, Hameed SM. Surgical fixation vs nonoperative management of flail chest: a meta-analysis. J Am Coll Surg. 2013;216(2):302-11.

18. Swart E, Laratta J, Slobogean G, Mehta S. Operative treatment of rib fractures in flail chest injuries: a meta-analysis and costeffectiveness analysis. J Orthop Trauma. 2017;31(2):64-70.

19. Pieracci FM, Majercik S, Ali-Osman F, Ang D, Doben A, Edwards JG, French B, Gasparri M, Marasco S, Minshall C, Sarani B, Tisol W, VanBoerum DH, White TW. Consensus statement: Surgical stabilization of rib fractures rib fracture colloquium clinical practice guidelines. Injury. 2017;48(2):307-21.

20. Kasotakis G, Hasenboehler EA, Streib EW, Patel N, Patel MB Alarcon L, Bosarge PL, Love J, Haut ER, Como JJ. Operative fixation of rib fractures after blunt trauma: a practice management guideline from the Eastern Association for the Surgery of Trauma. J Trauma Acute Care Surg. 2017;82(3):618-26.

21. DeFreest L, Tafen M, Bhakta A, Ata A, Martone S, Glotzer O, Krautsak K, Rosati C, Stain SC, Bonville D. Open reduction and internal fixation of rib fractures in polytrauma patients with flail chest. Am J Surg. 2016;211(4):761-7.

22. Farquhar J, Almahrabi Y, Slobogean G, Slobogean B, Garraway N, Simons RK, Hameed SM. No benefit to surgical fixation of flail chest injuries compared with modern comprehensive management: results of retrospective cohort study. Can J Surg. 2016;59(5):299-303. 
23. Holcomb JB, McMullin NR, Kozar RA, Lygas MH, Moore FA. Morbidity from rib fractures increases after age 45. J Am Coll Surg. 2003;196(4):549-55.

24. Campbell N, Conaglen P, Martin K, Antippa P. Surgical stabilization of rib fractures using Inion OTPS wraps-Techniques and quality of life follow-up. J Trauma. 2009;67(3):596-601.

25. Marasco SF, Davies AR, Cooper J, Varma D, Bennett V, Nevill R, Lee G, Bailey M, Fitzgerald M. Prospective randomized controlled trial of operative rib fixation in traumatic flail chest. J Am Coll Surg. 2013;216(5):924-32.

26. Granetzny A, El-Aal MA, Emam E, Shalaby A, Boseila A. Surgical versus conservative treatment of flail chest. Evaluation of the pulmonary status. Interact Cardiovasc Thorac Surg. 2005;4(6):583-7.

27. Sarani B, Schulte L, Diaz JJ. Pitfalls associated with open reduction and internal fixation of fractured ribs. Injury. 2015;46(12):2335-40.
28. Pieracci FM, Coleman J, Ali-Osman F, Mangram A, Majercik S, White TW, Jeremitsky E, Doben AR. A multicenter evaluation of the optimal timing of surgical stabilization of rib fractures. J Trauma Acute Care Surg. 2017. https://doi.org/10.1097/TA.00000 00000001729.

29. De Smet AM, Kluytmans JA, Blok HE, Mascini EM, Benus RF, Bernards AT, Kuijper EJ, Leverstein-van Hall MA, Jansz AR, de Jongh BM, van Asselt GJ, Frenay IH, Thijsen SF, Conijn SN, Kaan JA, Arends JP, Sturm PD, Bootsma MC, Bonten MJ. Selective digestive tract decontamination and selective oropharyngeal decontamination and antibiotic resistance in patients in intensivecare units: an open-label, clustered group-randomised, crossover study. Lancet Infect Dis. 2011;11(5):372-80.

30. Gardenbroek TJ, Bemelman M, Leenen LPH. Pseudoarthrosis of the ribs treated with a locking compression plate. J Bone Joint Surg Am. 2009;91(6):1477-9. 